\title{
Modelling the Soft X-Ray and EUV Emission in Classical Novae: $E U V E$ and ROSAT Observations of V1974 Cygni
}

\author{
GUY S. STRINGFELLOW ${ }^{1,2}$ AND STUART BOWYER ${ }^{1}$ \\ ${ }^{1}$ Center for EUV Astrophysics, University of California, 2150 Kittredge Street, \\ Berkeley, CA 94720-5030, USA
}

${ }^{2}$ Present Address: Department of Astronomy and Astrophysics, The Pennsylvania State University, 525 Davey Lab, University Park, PA 16802-6305, USA

We have conducted an extensive analysis of the observability of Classical Novae with the EUVE Lex/B and $\mathrm{Al} / \mathrm{Ti} / \mathrm{C}$ detectors. Predicted count rates have been computed using optically thin, isothermal plasma models for solar and metal-rich compositions, and hot $\mathrm{ONeMg}$ white dwarf model atmospheres. We find $E U V E$ to be quite sensitive to both the EUV and soft Xray emission emitted by the underlying hot white dwarf during novae outbursts, except for the coolest temperatures with very high intervening hydrogen column density. These results are used to interpret the emission detected during the EUVE all-sky survey of Nova Cygni 1992 (三 V1974 Cyg), 279-290 days after visual maximum. We find the best fit to the observed emission from V1974 Cyg arises from a hot $\mathrm{ONeMg}$ white dwarf with surface temperature $\sim 4 \times 10^{5}$ $\mathrm{K}$ and a mass of $\sim 1.2 M_{\odot}$, and derive an interstellar hydrogen column density of $\sim 3 \times 10^{21}$ $\mathrm{cm}^{-2}$. Virtually all this emission arises from supersoft X-rays rather than the EUV. We also report the detection of V1974 Cyg with the EUVE Deep Survey detector at 549 days after visual maximum. This observation is compatible with the above properties, indicating that the mechanism responsible for the soft X-ray emission, connected with the underlying white dwarf, had not yet entirely turned off. We also present analysis of a ROSAT PSPC observation which is contemporaneous with the $E U V E$ survey observations; this independently confirms the high column density we derived from the EUVE survey observation. Light curves for the EUVE and ROSAT observations are presented. Statistical tests for variability show that all of these observations are indeed highly variable over various time scales. The EUVE survey data shows one day variations, the EUVE DS data show $\sim 30$ minute fluctuations, while the ROSAT data vary rapidly on time scales of seconds. The EUVE data shows no periodic variability on any time scale. The implications of the rapid variability are briefly discussed.

\section{Introduction}

Nova Cygni 1992 (V1974 Cyg) was the optically brightest nova in nearly two decades, having been discovered on 19.1 Feb 1992 (Collins 1992), three days before reaching maximum visual brightness at $m_{V} \approx 4.4$ on $22.1 \mathrm{Feb}$. It was subsequently classified as an $\mathrm{ONeMg}$ nova (e.g., Hayward et al. 1992). We previously reported the detection of V1974 Cyg with the Extreme Ultraviolet Explorer (EUVE) during the all-sky survey (Stringfellow \& Bowyer 1993a,b), after the nova had entered its nebular stage at about 285 days after maximum visual brightness $\left(V_{\max }\right)$. A wide variety of distance estimates have been employed, yielding a range of 1.4-3.2 kpc (Chocol et al. 1993; Stringfellow \& Bowyer 1994; Paresce et al. 1995; and references therein), which suggests the interstellar hydrogen column density, $N_{\mathrm{H}}$, should be $\geq 10^{21} \mathrm{~cm}^{-2}$. In this paper we show that the $E U V E$ detection arises predominantly from supersoft $\mathrm{X}$-rays $(\lambda<44 \AA$ ), in a regime where the $E U V E$ effective area curves have less sensitivity, but where they are nonetheless still capable of detecting very bright sources. The emission detected during the all-sky sur- 
vey is best described by radiation emitted by a white dwarf with surface temperature $\sim 400000 \mathrm{~K}$, and $N_{\mathrm{H}} \sim 3 \times 10^{21}$. This conclusion is in general agreement with the analysis of ROSAT observations during the EUVE survey observation, an example of which is discussed below. EUVE and ROSAT observations during Nov-Dec 1992 are analyzed for variability, along with a subsequent $E U V E$ pointed observation made with the Deep Survey detector 549 days after $V_{\max }$. We find all these lightcurves to be highly variable.

\section{Modelling the $E U V E$ Survey Observations}

Stringfellow \& Bowyer (1993ab, 1994) reported detection of V1974 Cyg by the EUVE during the course of the all-sky survey, scanned between $27 \mathrm{Nov}$ and $8 \mathrm{Dec} 1992$. The mean count rates in the Lex/B and $\mathrm{Al} / \mathrm{Ti} / \mathrm{C}$ filters are $0.112 \pm 0.010$ counts $^{-1}$ and $0.102 \pm 0.014$ counts $\mathrm{s}^{-1}$, corresponding to $18 \sigma$ and $9 \sigma$ detections, respectively. The $E U V E$ scanner effective areas are sensitive down into the supersoft $\mathrm{X}$-ray domain and clearly the flux detectable in both supersoft X-rays and EUV depends critically on the precise value of $N_{\mathrm{H}}$.

Several potential emission mechanisms could be operating in V1974 Cyg simultaneously. The clumpy nova ejecta model discussed by Williams (1992) and Saizar \& Ferland (1994) is illustrative of the physically distinct components and their interactions, although the properties of each component could vary widely in each nova, and indeed during the evolution of any given nova. A general composite model will contain the hot $\left(>10^{5} \mathrm{~K}\right)$ underlying white dwarf, while the ejecta contains warm $\left(10^{4}-10^{5} \mathrm{~K}\right)$ clumps and a hot $\left(10^{6} \mathrm{~K}\right)$, interclump plasma filling a large volume. Shocks arising through interactions with various components may also exist, although perhaps only during specific evolutionary stages during the outburst. Although there have been no reported observations indicating the presence of dust in the ejecta of V1974 Cyg thus far, another fast ONeMg nova, Nova Her 1991 ( $\equiv$ V838 Her), has been shown to form dust early, within a few days after outburst (Harrison \& Stringfellow 1994). Obviously, each nova is unique, even within a specific class, and their ejecta environment are complex and variable.

We have computed an extensive grid of isothermal, optically thin plasma models for both solar and metal-rich compositions, the latter representative of the ejecta of $\mathrm{ONeMg}$ novae. Generally speaking, these models alone are unable to account for the observed EUVE emission as the dominant component. Hence, we forgo discussing details of these models here; early results were presented by Stringfellow \& Bowyer (1994), and full details of these and the white dwarf modelling will appear elsewhere.

Figure 1 shows our derived $E U V E$ count rates for a $1.2 M_{\odot} \mathrm{ONeMg}$ white dwarf with surface temperatures ranging from $10^{5}-10^{6} \mathrm{~K}$ (MacDonald \& Vennes 1991). The models span the wavelength range from $10-300 \AA$. The curves correspond to various values of $N_{\mathrm{H}}$ at a distance of $1 \mathrm{kpc}$, but these can be scaled simply to any distance. Note the $E U V E$ count rates are generally quite high, implying that the hottest $\mathrm{ONeMg}$ novae with massive white dwarfs are easily detectable by $E U V E$ out to large distances and high $N_{\mathrm{H}}$. Observability of the coolest $\mathrm{ONeMg}$ novae $\left(\sim\right.$ few $\left.\times 10^{5} \mathrm{~K}\right)$ are most strongly dependent upon the intervening $N_{\mathbf{H}}$. The two horizontal lines in Figure 1 are the fitted count rates of the models at $1.4 \mathrm{kpc}$ (lower line) and $3.0 \mathrm{kpc}$ (upper line), which bound the range of permitted models for the range in derived distances to V1974 Cyg.

The observed $E U V E$ count rates imply a white dwarf temperature between $3-5 \times 10^{5} \mathrm{~K}$, whereas the modelled ratio of the count rates in the two filters demands that $N_{\mathrm{H}}>10^{21}$ $\mathrm{cm}^{-2}$; at $N_{\mathrm{H}}=3 \times 10^{21} \mathrm{~cm}^{-2}$, the ratio of the $\mathrm{Al} / \mathrm{Ti} / \mathrm{C}$ to Lex/B count rates become insensitive (i.e., flat) to the WD effective temperature, and lower than the observed ratio by about a factor of 4 . This simply reflects the ratio of the effective area curves of the 


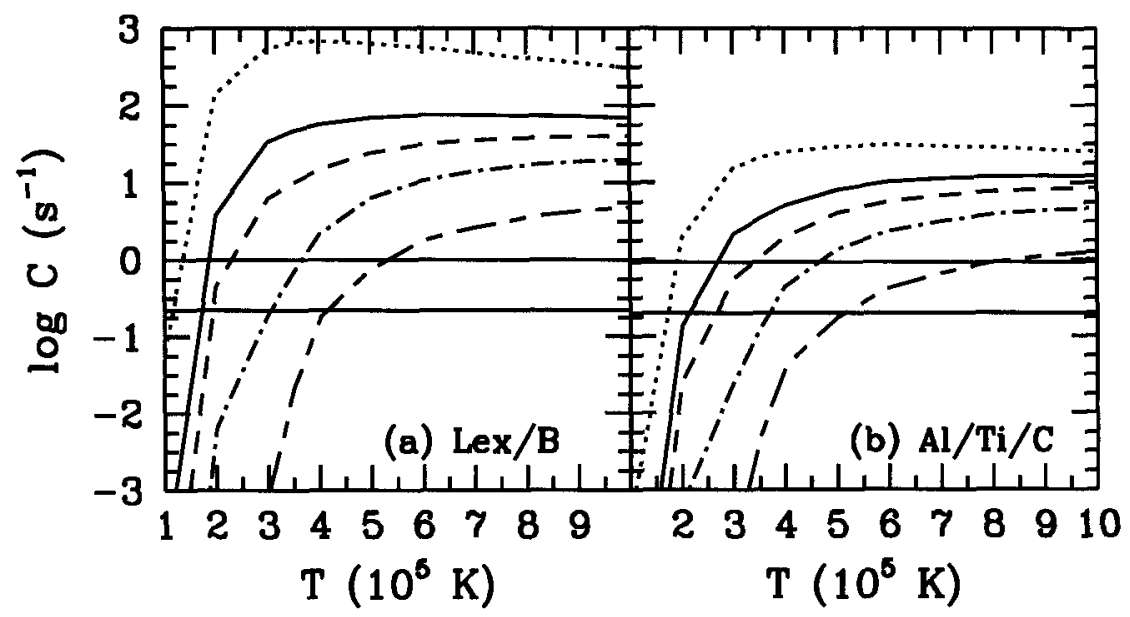

Figure 1. Computed $E U V E$ count rates for a $1.2 M_{\odot}$ ONeMg white dwarf at 1 kpc. The various curves are the computed count rates for model white dwarfs with corresponding surface temperatures attenuated by the ISM for values of $\log N_{\mathrm{H}}$ of $21.5,21.0,20.7,20.5$, and 20.0 $\mathrm{cm}^{-2}$ from the bottom up, respectively. The $E U V E$ filters employed in the computations of the model count rates are noted in each panel. The two horizontal lines correspond to the $E U V E$ observed count rates for V1974 Cyg in each filter if it lies at a distance of $1.4 \mathrm{kpc}$ (lower) or 3 kpc (upper).

two filters below $44 \AA$ (the last wavelength at which ground based calibrations exist for both filters). The Lex/B filter effective area curve has been adjusted in this regime to match the EUVE survey count rate observed for Sco X-1, using its well known X-ray modelled parameters, but no such secondary calibration has yet been performed for the $\mathrm{Al} / \mathrm{Ti} / \mathrm{C}$ filter. Therefore, we attribute the factor of 4 discrepancy between the modelled and observed count rate in the $\mathrm{Al} / \mathrm{Ti} / \mathrm{C}$ filter to this 'secondary calibration' problem. Inspection of the effective area curves for the two filters indicates that the $\mathrm{Al} / \mathrm{Ti} / \mathrm{C}$ count rate should always be at least a factor of $\sim 10$ lower than that in the Lex/B filter between $44-160 \AA$. Al/Ti/C-to-Lex/B count rate ratios near unity are permitted only for sources with EUV-dominated flux in the narrow range $160-180 \AA$, or according to our analysis presented herein, when the radiation arises below $44 \AA$. Thus, we conclude from the analysis of the $E U V E$ survey data that essentially all of the emission observed from V1974 Cyg arises from supersoft X-rays. Note that the observed count rate ratio for Sco X-1 is in accord with the above arguments. Analysis of EUVE sources with observed $A l / T i / C$-to-Lex $/ B$ count rate ratios greater than 0.1 could yield a population of (previously unidentified?) supersoft $X$-ray candidates.

\section{Rapid Soft X-ray Variability in V1974 Cyg}

The EUVE survey light curve for the Lex/B filter is shown in Figure 2a. The count rates correspond to actual scanned times over $12^{\mathrm{h}}$ time intervals. We also report for the first time the EUVE detection of V1974 Cyg with the Deep Survey detector (DS) during a $\sim 38$ ks pointed observation in August 1993, 549 days after $V_{\text {max }}$. This latter light curve is displayed in Figure 2b. The DS data have been corrected for the loss of sensitivity resulting from lying on the detector "dead spot." We have performed a chi- 


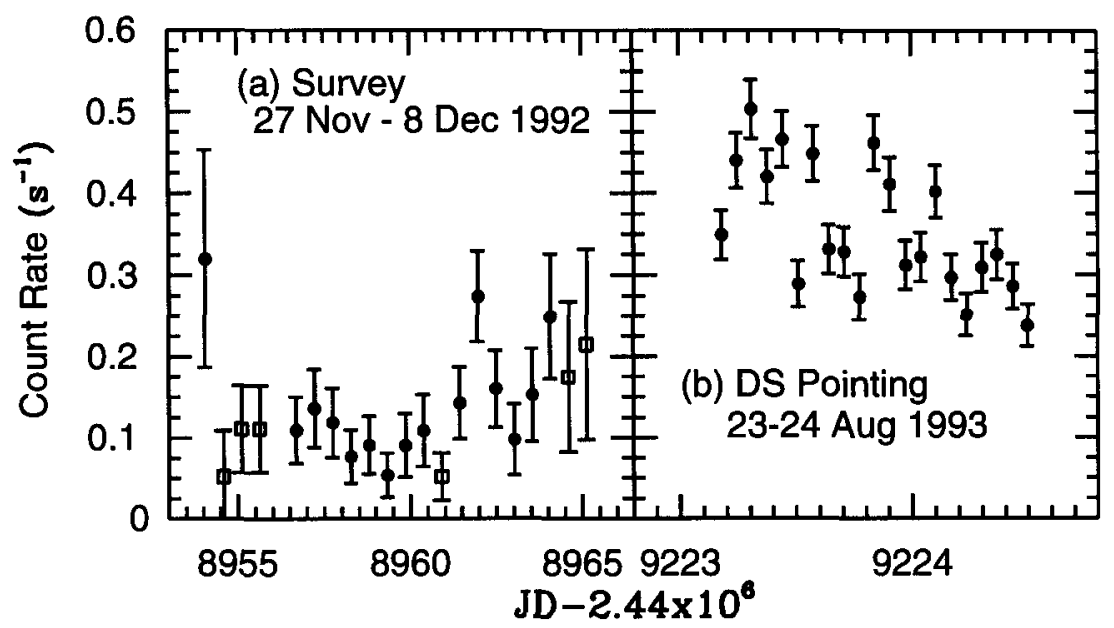

FIGURE 2. Lightcurves of V1974 Cyg observed by EUVE during (a) the Lex/B filter during the all-sky survey, where the open squares denote $<3 \sigma$ significance, and (b) a pointed observation with the Deep Survey detector. Each survey bin represents the actual scanned time during a 12 hour interval (typically $\sim 100 \pm 50 \mathrm{~s}$ ), whereas the DS data are the continuous pointed time per $E U V E$ orbit (roughly $30.8 \mathrm{~min}$ ).

square analysis on both data sets and find each to be highly variable, with confidence levels of $99 \%$ for the survey data and $\gg 99.9 \%$ for the DS data.

ROSAT has followed the temporal evolution of V1974 Cyg at irregular intervals with the PSPC, starting at 60 days after $V_{\max }$. One such observation occurred on 6 Dec 1992, 288 days after $V_{\max }$, which overlaps with the observational period during the EUVE survey data; it corresponds to a low-state in the survey light curve (fifth point from the left in Fig. 2a). This lightcurve is shown in Figure 3, where the data have been barycenter corrected, and binned into $10 \mathrm{~s}$ intervals. While the mean count rate over this continuous observation is $31.4 \mathrm{~s}^{-1}$, visual inspection clearly shows rapid fluctuations by as much as a factor of two in less than $20 \mathrm{~s}$. The data span a range of $18-55$ counts $\mathrm{s}^{-1}$. We have performed a Kolmogorov-Smirnov (KS) and Cramer-von Mises statistical tests, and find the data to be inconsistent with a constant source intensity at an extremely high confidence level, $\gg 99.9 \%$. Other ROSAT PSPC data sets we have analyzed also exhibit such behavior as that in Figure 3. Discovery of such rapid variability, on time scales of seconds, implies (a) a compact region for the source of the supersoft X-rays, and (b) undoubtedly accretion instabilities are responsible. Whether this corresponds to an accretion stream/hotspot or related instead to reformation of an accretion disk, is presently unclear and requires further analysis which is presently underway. It may even be possible to account for the rapid $X$-ray variability by invoking clumpy accretion of the remaining ejecta which did not reach, or maintain, escape velocity; this material could be "raining" back onto the white dwarf surface. The concept of stable (i.e., constant) thermonuclear burning on the white dwarf surface requires review in light of these results.

We wish to thank the CEA science and DASS teams for useful discussions and support, Martin Sirk for assistance with the EUVE analysis software, and Stephane Vennes for providing the white dwarf atmosphere models. This research has been supported in part by NASA contract NAS5-30180. 


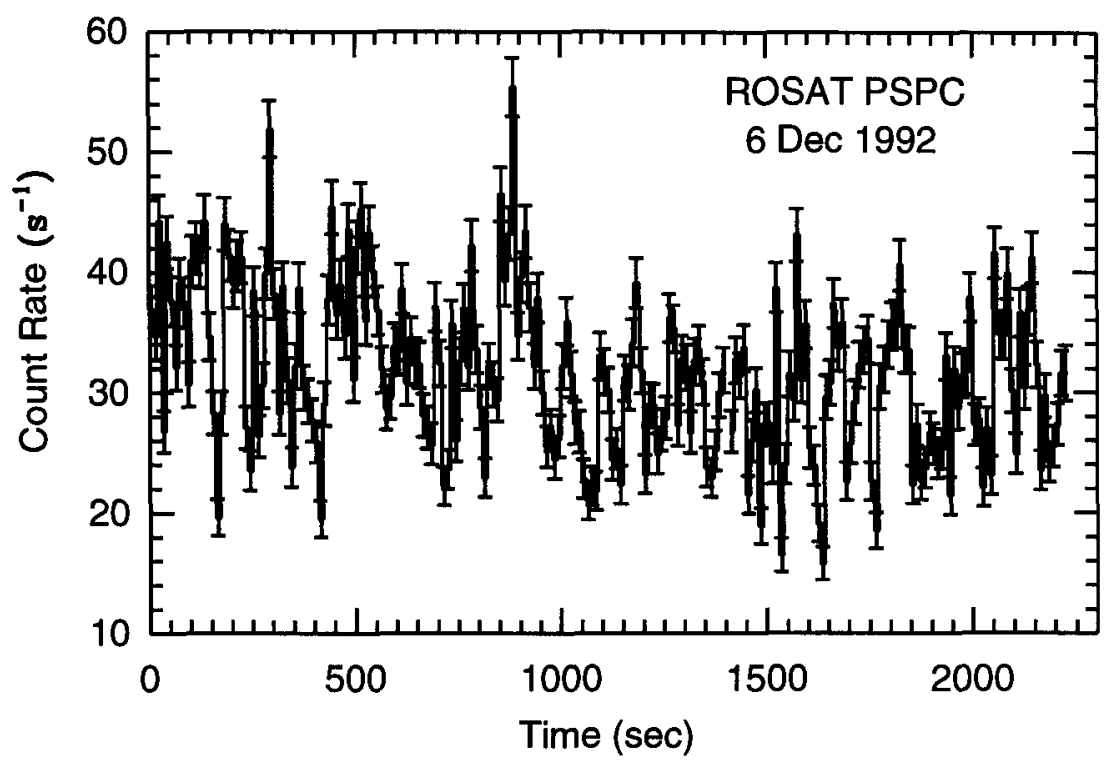

FIgURE 3. ROSAT PSPC lightcurve of V1974 Cyg on 6 Dec 1992. The start of the observation corresponds to JD $=2448962.9360987$. The data have been binned into $10 \mathrm{~s}$ intervals, and are clearly highly variable.

\section{REFERENCES}

Collins, P. 1992, IAU Circular No. 5454

Chocol, D., Hric, L., Urban, Z., KomziK, R., Grygar, J., \& PapouseK, J. 1993, A\&A, 277,103

Harrison, T. E. \& Stringfellow, G. S. 1994, ApJ, 437, 827

Hayward, T. L., GehrTz, R. D., MLES, J. W., \& HouCK, J. R. 1992, ApJ, 401, L101

MacDonald, J. \& Vennes, S. 1991, ApJ, 373, L51

Paresce, F., Livio, M., Hack, W., \& Korista, K. 1995, A\&A, in press

Saizar, P. \& Ferland, G. J. 1994, ApJ, 425, 755

Stringfellow, G. S. \& BowYeR, S. 1993a, IAU Circular No. 5803

STRINGFELlOW, G. S. \& BOWYER, S. 1993b, BAAS, 25, 1250

Stringfellow, G. S. \& Bowyer, S. 1994, In Interacting Binary Stars, ed. A. W. Shafter, ASP Conference Series, 37, 315

Williams, R. E. 1991, ApJ, 392, 99 